\title{
MALADAPTIVE COGNITIONS, HYPERACTIVITY AND INCONSISTENT PARENTING AS RISK FACTORS OF ADOLESCENT PROBLEMATIC INTERNET USE
}

\begin{abstract}
The aim of this study was to examine the interrelationships of specific adolescent biological, environmental (parental), and cognitive factors in relation to adolescent problematic internet use (PIU). The design of the present study was influenced by the theoretical framework presented by Brand and colleagues (Brand, et al., 2016), who have proposed a multi-faceted model, including a vast number of interacting variables. This study was with the intent of focusing upon three possible risk factors suggested by this model. Participating in the study were 307 middle-school and high-school adolescents, who completed questionnaires regarding hyperactivity, parenting practices, and maladaptive cognitions. After controlling for age, gender and family economic status, regression analysis showed hyperactivity, inconsistent parenting, and maladaptive cognitions to be predictive of PIU. Maladaptive cognitions were shown to partially mediate the effect between insonsistent parenting and PIU. Results are discussed in regard to the necessity of considering multiple prevention and treatment approaches in light of the various PIU risk factors within the neuropsychological, familial and cognitive domains.
\end{abstract}

Keywords: Problematic internet use, Hyperactivity, Parenting practices, Maladaptive cognitions.

\section{Introduction}

Internet use is becomming an ever more increasing and almost indispensable part of an adolescent's daily life. This includes the necessity of using the internet for academic purposes in completing school assignments, but also for daily communication with peers and parents. As a result of the 
increased use of the internet, the risks of internet overuse and development of internet dependency are augmented. Recently researchers of internet use (Brand, Young, \& Laier, 2014; Brand, et al., 2016; Davis, 2001) have developed several models depicting an integration of internet dependency risk factors, including neurobiological, experiential, affective and cognitive components contributing to internet use disorders, and these models have helped to provide a theoretical framework for the present research study.

Two decades have passed since Kimberly Young (Young, 1998) began to examine the potential psychological dangers and the severe psychosocial problems which can result from excessive internet use. Since this initial acknowledgment of the potential psychological difficulties associated with intensified internet use, various terms have been applied to these difficulties, including Internet addiction, Internet addiction disorder, Problematic internet use (PIU), Pathological internet use, and Compulsive internet use (Ciarrochi, et al., 2016). In general, these terms similarly refer to an inability to exert control over one's use of the internet, with subsequent negative consequences for meaningful daily activities. Internet addiction or Problematic internet use shares similar symptoms with other addictive disorders, including preoccupation, uncontrolled impulse to engage, withdrawal phenomena, tolerance, excessive time and effort devoted to the addictive behavior, and negative social repercussions (Ko, et al., 2007; Pies, 2009).

The relationship between adolescent problematic internet use and predictive, concurrent or subsequent socioemotional difficulty has been examined in many parts of the world, with the use of both cross-sectional and longitudinal studies. In general, associations have been found between intensified, problematic internet use and a wide range of indices of mental health, including lowered self-esteem, loneliness, depression, anxiety and social phobia (Park, et al., 2008; Selfhout, et al., 2009). A cross-sectional study examining PIU in association with psychological impairments among European adolescents showed that suicidal behaviors (suicidal ideation and suicide attempts), depression, anxiety, conduct problems and hyperactivity/inattention were significant and independent predictors of PIU (Kaess, et al., 2014).

Previous studies of adolescent Problematic Internet use (PIU) in Latvia have shown direct associations with aggression, as well as associations with anxiety, dissociation and sexual concerns (Miltuze, Bite, \& Sebre, 2012). In Lithuania adolescent Problematic internet use (PIU) was found to be associated with negative emotionality and less satisfaction in friendships and familial relationships (Jusiene, Cesniene, \& Mordas, 2015). As a significant risk factor for the development of Internet addiction, Ko and colleagues have accentuated the importance of the amount of time spend in online gaming (Ko, et al., 2007). 


\section{Hyperactivity as a risk factor for PIU}

Hyperactivity has been linked to internet addiction in several previous studies, as indicated by meta-analyses investigating this association (Carli, 2013; Wang, et al., 2017). It has been shown that adolescents diagnosed with attention deficit hyperactivity disorder (ADHD) were more likely to be addicted to the internet than those with other difficulties such as agression or social phobia. Researchers have suggested that since one of the symptoms of ADHD is "being easily bored", the internet use may serve to alleviate feelings of boredom because it is possible to play several games simultaneously, or participate in several chat conversations within the same time frame. Another symptom of ADHD is increased impulsivity, and having an aversion for delayed rewards. This may facilitate internet addiction because the internet provides opportunity for receiving immediate stimulation and immediate rewards, such as game points. Also, as indicated by Brand and colleagues (Brand, et al., 2014; Brand, et al., 2016), differences in the prefrontal lobe brain activity of individuals with ADHD are linked to impaired inhibition and lack of self-control, which would make it more difficult for individuals with hyperactivity to stop using the internet once they have begun. Hyperactivity is largely considered to be genetically influenced, with genetic factors accounting for $73 \%$ of the variability in hyperactivity, as shown in a recent meta-analysis (Nikolas \& Burt, 2010).

\section{Parenting practices as a risk factors for PIU}

Parenting practices and other aspects of family functioning have been found to be associated with problematic internet use in numerous studies (e.g., Ko, et al., 2007; Yen, et al., 2007), and the results of these studies have indicated that higher parent-adolescent conflict, habitual alcohol use by siblings, and perceived parents' negative attitude toward adolescent substance use is predictive of PIU. Low family functioning and increased amount of online gaming has predicted the emergence of internet addiction. In contrast, emotionally supportive parenting style has been found to be predictive of a lower likelihood of internet addiction (Chen, Chen \& Gau, 2015; Park, Kim, \& Cho, 2008; Van den Eijenden, 2010). Negative experiences in the parent-child relationship have been linked to insecure attachment style, and insecure attachment has been found to be associated with internet use disorders (Schimmenti, et al., 2014). It has consistently been shown that emotionally warm and supportive parenting practices facilitate child mental health, whereas punishment oriented practices facilitate child internalizing and externalizing problems (e.g. Sebre, et al., 2014). In addition, inconsistent disciplining, when discipline 
may erratically fluctuate depending upon the parents' mood or the child's powers of persuasion, has also shown to directly contribute to child mental health problems and delinquent-oriented attitudes (Balan, et al., 2017; Halgunseth, et al., 2013).

\section{Maladaptive cognitions as risk factors for PIU}

Maladaptive cognitions have been linked to Problematic internet use in a theoretical model based upon a cognitive-behavioral approach. Davis (2001) has proposed that maladaptive cognitions can serve as a "main source" of maladaptive behavior, and can encourage maladaptive behaviors such as PIU. Davis frames his consideration of the role of maladaptive cognitions within the diathesis-stress model, whereby the individual's predisposing vulnerability is accelerated within stressful life situations. Specifically, Davis proposes that if a predisposing vulnerability such as depression or anxiety is coupled with a stressful situation such as lack of support from family and friends, these can be facilitating factors which thereby promote maladaptive cognitions. Similar to the types of negative cognitions proposed by cognitive-behaviouralists Ehlers \& Clark (2000), Davis considers the distinction between negative thoughts about the self, and negative thoughts about the world. Predisposing socioemotional factors, in conjunction with maladaptive cognitions, can facilitate overuse of the internet. This may be further augmented in situations where the internet user experiences positive reinforcement, for example, temporary relief from anxiety, and a fulfillment of internet-related expectations, such as being able to "make friends" more easily. The role of cognition, specifically agressive cognitions, has also been accentuated by Anderson and colleagues (Anderson, et al., 2017), who have found that the association between violent video-gaming and aggression is partially mediated by aggressive cognitions.

Integrated model of biological, social and cognitive risks factors for PIU

Several of the leading scientists concerned with internet addiction (e.g., Brand, Young, \& Laier, 2014) have acknowledged the significance of the cognitive-behavioral model proposed by Davis, but have elaborated upon this model by including neuropsychological mechanisms, specifically the control processes mediated by prefrontal brain areas. During the past decade there have been numerous studies linking PIU with executive functions, for example, difficulties in inhibiting automatic responses and difficulties with mental flexibility, i.e. set-shifting (Zhou, et al., 2012). Studies employing 
functional magnetic resonance imaging (e.g. Dong, et al., 2013) have shown that patients with internet addiction have reductions in decision-making performance, and this may contribute to their continuation of game playing even though they are confronted with negative consequences. Brand and colleagues (Brand, Young, \& Laier, 2014) have concluded that individuals with internet addiction may have reduced effectiveness of the prefrontal control processes, and this may be related to their loss of control over how many hours they spend engaged with the internet.

Brand has now expanded his team of collaborators (Brand, et al., 2016), and they have elaborated the theoretical framework in proposing the Interaction of Person-Affect-Cognition-Execution (I-PACE) model. This model includes several of the components initially proposed by

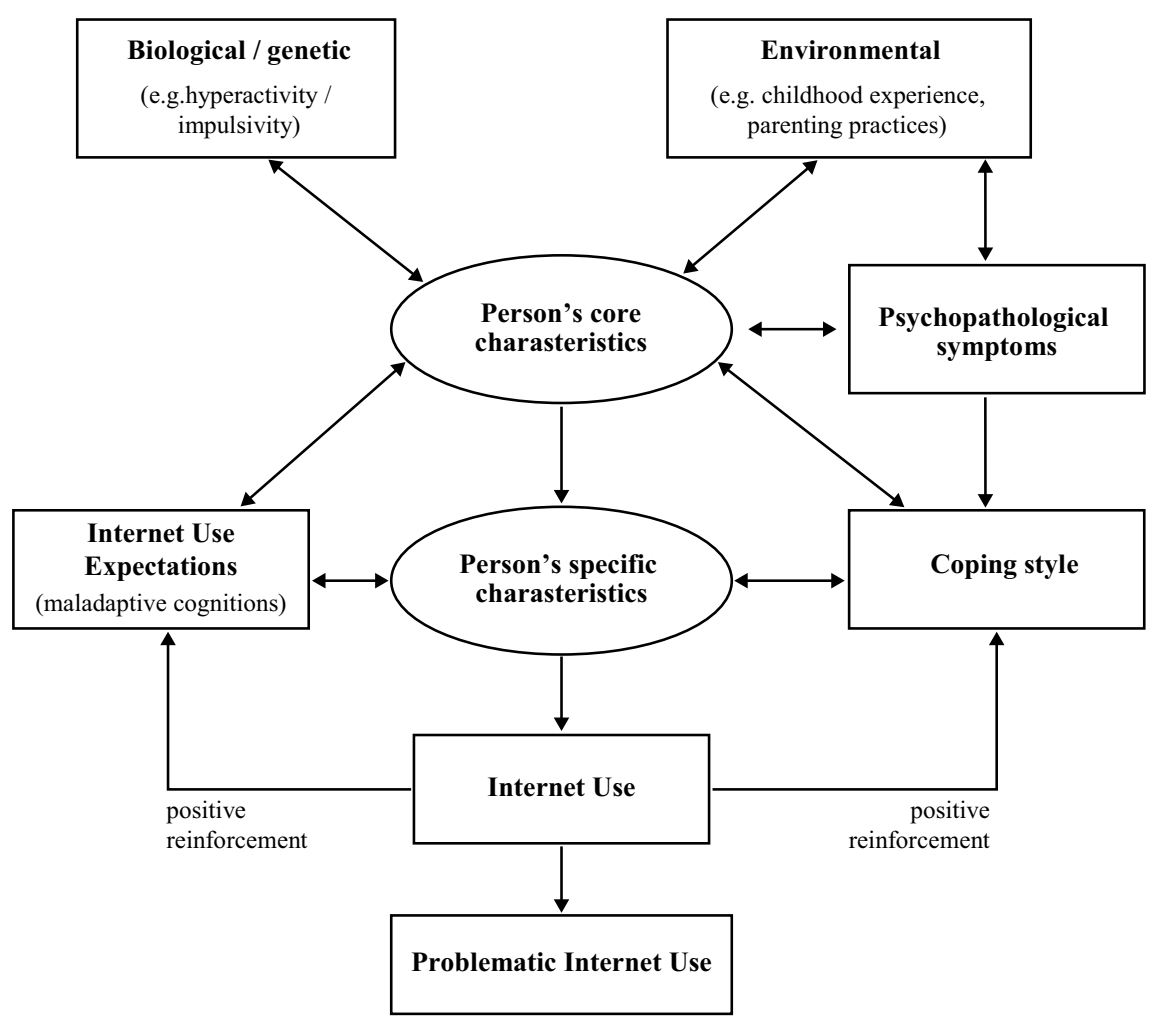

Figure 1. Theoretical model, based upon the models of Brand and colleagues (Brand, Young, \& Laier, 2014; Brand, et al., 2016) of interrelationships of biological, early environmental, psychopathological symptoms, coping style and maldaptive cognitions as facilitating Problematic Internet Use. The variable addressed int the present study are hyperactivity, parenting practices and maladaptive cognitions. 
Davis (2001), such as biopsychological vulnerability factors, including depression and anxiety disorders, as well as early childhood experiences and insecurity of attachment. The I-PACE model also incorporates internetrelated cognitive biases or maladaptive expectations which can promote internet addiction, as well as the coping styles by which a person attempts to deal with stressful situations. In addition, the I-PACE model includes neuropsychological mechanisms and executive control functions such as difficulties with inhibition and impulsivity as seen in attention-deficit/ hyperacitivity disorder (ADHD), one of the identified co-morbid conditions of internet-use disorders (Ho, et al., 2014). Brand and colleagues conclude with a call for additional research, specifically in investigating the interactions of these variables instead of studying them separately. Already Zhang, Li and Li (2015) have investigated several interactions of the above noted risk factors and found that maladaptive cognitions mediated the relation between temperament (e.g. effortful control and sensation seeking) and PIU, and that this relation was moderated by parenting styles.

Few studies to date have examined simultaneously biological, social and cognitive aspects as predictors of PIU. The present study is with the goal of examining the interrelationships between several potential risk factors of PIU, including possible "biopsychological contributions" (Brand, et al., 2016) such as hyperactivity and parenting practices, as well as internetrelated maladaptive cognitions. We hypothesize that adolescent reported hyperactivity, unsupportive parenting practices, as well as internet-related maladaptive cognitions will be predictive of PIU. We also hypothesize maladaptive cognitions to partially mediate the effect between parenting practices and PIU.

\section{Method}

\section{Participants}

Participants of the study were 307 adolescents (185 girls and 122 boys) aged between 13 to 19 years $(M=15.96$; $S D=2.45)$. The study participants were in attendance at middle school or high school, in classes from $7^{\text {th }}$ through $12^{\text {th }}$ grade. The participants were from various regions of Latvia, including both large and middle-sized cities. Schools were chosen according to accesibility. Adolescents reported their ethnicity as follows: $93.1 \%$ Latvian, $6.2 \%$ Russian, $0.7 \%$ other. Almost all of the study participants reported that computers and smart phones are readily available to them: $97 \%$ reported that they have a computer at home; $98 \%$ reported that they have internet access at home; 96\% reported that they have their own smart phone with internet access. 


\section{Procedure}

Permission was received from the school principals to conduct the study. Parents were informed, and passive consent was received, meaning that the parents were advised to indicate if they did not want their child to participate. Students were informed about the purpose of the study, principles of anonymity and voluntary participation. Active consent was received from all of the study participants. Questionnaires were distributed and collected by trained research assistants during homeroom class sessions.

\section{Measures}

All measures were forward and back-translated from English to Latvian and back by bilingual translators, with each translation performed independently. Consensus agreement was reached in regard to any discrepancies between the translations. The agreed upon translation was then pilot-tested and after initial psychometric analysis, including reliability and factor analysis, some items were relocated from one subscale to another. In some other cases the translation was made more precise in order to ensure conceptual equivalence of the items in comparison to the original. The questionnaire format was universally adapted to a 5-point Likert-type scale in order to facilitate the adolescents' familiarity and ease with the questionnaire format.

\section{Hyperactivity}

Hyperactivity was assessed with the hyperactivity subscale of the adolescent self-report form of the Strengths and Difficulties Questionnaire (SDQ; Goodman, 1997). The hyperactivity subscale has 5 items (e.g. "I am constantly fidgeting or squirming", and "I think before I do things", to be coded in reverse). The adolescents were asked to rate each item on a 5-point Likert-type scale from 0 ("never") to 4 ("always"). Cronbach's alpha rating for this scale was 71 in the present study.

\section{Parenting practices}

Parenting practices were assessed with several subscales from the Alabama Parenting Questionnaire (APQ; Frick, 1991; Shelton, Frick, \& Wootton, 1996): positive parenting, inconsistent discipline, and harsh punishment. The questionnaire completed by the adolescents included 11 items from the positive parenting subscale (e.g., "My parents have friendly talks with me"); seven items from the inconsistent discipline 
subscale (e.g., "My parents threaten to punish me but then actually do not punish me"); and four items concerning harsh punishment, adapted to their childhood experience (e.g., "When I was a child my parents spanked me when I had done something wrong"). All items were rated on a 5-point Likert-type scale from 0 ("never") to 4 ("always"). Cronbach's alpha ratings were from .67 to .84 in the present study.

\section{Maladaptive cognitions}

In this study the construct of maladaptive cognitions was considered as stemming from often unrealistic expectations or exagerated believs which favor the online world. In this study adolescents responded to 9 items, similar to those used by Li, et al. (2010), and Zhang, Li and Li (2015). Items such as "Friends online are more trustable than those offline" implies a negative cognition of others in the real world as untrustable, and the expectation that others who are online can be trusted. Items such as "When I am online I don't have do think about my problems related to school assignments" implies a distrust in one's own abilities to deal with one's school problems in a more adaptive way, such as completing one's assignments, but rather in dealing with school problems by escaping in the internet. The 9 maladaptive cognitions were rated on a 5-point Likerttype scale from 1 ("totally disagree") to 5 ("totally agree"). The Cronbach's alpha was .81 for the present sample.

\section{Problematic internet use}

The Problematic Internet Use scale was developed specifically for this study, based upon the Compulsive Internet Use Scale (Meerkerk, et al., 2009). This brief self-report measure included 4 items involving diagnostic criteria for problematic internet use: preoccupation with using the internet; need to use increased amounts of time; restless, moody or depressed when not using the internet; using the internet as way of escaping from problems. Items were rated on a 5-point scale, ranging from 1 ("never") to 5 ("always"). Cronbach's alpha was .78 for the present sample.

\section{Family economic status}

The adolescent's perception of family economic status was measured by asking the adolescent to mark one of the following: "my family can afford all that we want"; "my family is fairly well off"; "we get along at an average level"; "my family can afford only basic necessities"; "we are lacking even in the basic necessities". 


\section{Data Analysis}

Statistical analyses were conducted using SPSS version 22. First, zeroorder correlation coefficients were calculated to examine bivariate associations among the ratings of hyperactivity, parenting practices, maladaptive cognitions and PIU. Second, regression analyses were conducted, controlling for age, gender and family economic situation entered in the first step, in order to examine the predictive effects of hyperactivity, maladaptive cognitions, and which of the three measured parenting practices (positive parenting, harsh punishment and inconsistent discipline) would be shown to be significant predictors of PIU, when all four ratings were simultaneously entered in the second step. Third, the significant independent variables from the above regression analyses were entered into a combined regression model, again with age, gender and economic status entered in the first step. Fourth, the possibility of a mediation effect, as seen in the combined regression model was tested with an additional series of four regression analyses, all of which included the control variables age, gender and family economic status: PIU was regressed on maladaptive cognitions and inconsistent parenting; maladaptive cognitions were regressed on inconsistent parenting: and PIU was regressed on inconsistent parenting, accounting for the mediator maladaptive cognitions.

\section{Results}

Descriptive statistics and bivariate associations between the study variables are presented in Table 1. Problematic internet use was found to be positively correlated with hyperactivity, harsh parenting, inconsistent parenting, as well as maladaptive cognitions. Maladaptive cognitions were negatively correlated with positive parenting, but positively correlated with hyperactivity and inconsistent parenting.

When analyzing the two parenting practices (harsh discipline and inconsistent parenting) which had been shown to correlated positively with PIU, initial regression analysis showed inconsistent discipline to remain as the only significant predictor of PIU, when controlling for age, gender and family economic status, $\mathrm{F}(5,290)=5.450, p<.001$. The main regression model was computed in order to examine the combined effects of a biological factor (hyperactivity), social factor (inconsistent parenting) and cognitive factor (maladaptive cognitions), while controlling for age, gender and family economic status in the first step, in prediction of PIU. The results showed hyperactivity to be a significant contributing factor in the second step. In the third step both hyperactivity and inconsistent discipline were shown to be independent contributing factors to the variation in 
PIU ratings. However, with the addition of maladaptive cognitions ratings in the fourth step, there was a change in the Beta value of inconsistent discipline ratings (from $.20, p<.01$ to $.10, p=.079$ ), while hyperactivity and maladaptive cognitions were shown to be predictive of PIU ratings.

Table 1. Descriptive statistics and zero-order bivariante correlations between study variables

\begin{tabular}{lcccccc}
\hline & $\mathbf{1}$ & $\mathbf{2}$ & $\mathbf{3}$ & $\mathbf{4}$ & $\mathbf{5}$ & $\mathbf{6}$ \\
\hline 1. Problematic internet use & & $.31^{* * *}$ & -.06 & $.12^{*}$ & $.25^{* * *}$ & $.44^{* * *}$ \\
2. Hyperactivity & & & $-.26^{* * *}$ & .10 & $.29^{* * *}$ & $.19^{* *}$ \\
3. Positive parenting & & & & $-.26^{* * *}$ & $-.14^{*}$ & $-.12^{*}$ \\
4. Harsh punishment & & & & & $.29 * * *$ & .07 \\
5. Inconsistent parenting & & & & & & $.26^{* * *}$ \\
6. Maladaptive cognitions & & & & & & \\
Mean & 2.20 & 2.56 & 3.75 & 1.62 & 2.15 & 1.99 \\
SD & .79 & .68 & .80 & .77 & .71 & .71 \\
\hline
\end{tabular}

$* p<.05, * * p<.01, * * * p<.001$

Table 2. Hierarchical regression analyses including age, gender, material well-being, hyperactivity, inconsistent discipline and maladaptive cognitions predicting Problematic Internet Use $(N=305)$

\begin{tabular}{lcccccc}
\hline Predictor variable & & $\boldsymbol{B}$ & $\boldsymbol{S E}$ & $\boldsymbol{\beta}$ & $\boldsymbol{p}$ & $\boldsymbol{R}^{2}$ \\
\hline Step 1 & & & & & & .01 \\
& Age & -.02 & .02 & -.05 & .44 & \\
& Gender & .06 & .10 & .03 & .56 & \\
\hline Step 2 & .12 & .07 & .10 & .09 & \\
& Material well-being & & & & & .10 \\
& Age & -.02 & .02 & -.06 & .33 & \\
& Gender & -.03 & .09 & -.02 & .78 & \\
& Material well-being & .13 & .07 & .11 & .06 & \\
Step 3 & .35 & .07 & $.30 * * *$ & .00 & \\
& Hyperactivity & & & & & .14 \\
& Age & -.02 & .09 & -.06 & .27 & \\
& Gender & -.06 & .09 & -.04 & .49 & \\
& Material well-being & .10 & .07 & .08 & .13 & \\
& Hyperactivity & .28 & .07 & $.24 * * *$ & .00 & \\
& Inconsistent discipline & .26 & .08 & $.20 * *$ & .00 & \\
\hline
\end{tabular}




\begin{tabular}{lccccc}
\hline Predictor variable & $\boldsymbol{B}$ & $\boldsymbol{S E}$ & $\boldsymbol{\beta}$ & $\boldsymbol{p}$ & $\boldsymbol{R}^{2}$ \\
\hline Step 4 & & & & & .26 \\
Age & .01 & .02 & .02 & .69 & \\
Gender & -.08 & .08 & -.05 & .32 & \\
Material well-being & .08 & .06 & .07 & .20 & \\
Hyperactivity & .24 & .06 & $.21 * * *$ & .00 & \\
Inconsistent discipline & .13 & .07 & .10 & .08 & \\
Maladaptive cognitions & .42 & .06 & $.38 * * *$ & .00 & \\
\hline
\end{tabular}

$* * p<.01 ; * * * p<.001 ; \mathrm{F}(6,289)=16.509, p<.001$.

A final examination of mediation affects showed there to be a partial mediation effect of maladaptive cognitions between inconsistent parenting and PIU (see Table 3). Again all regressions were calculated controlling for age, gender and family economic status in the first step. Results showed a significant direct relation between inconsistent parenting and PIU $(\beta=.28$, $S E=.07)$, and a significant direct relation between maladaptive cognitions and PIU $(\beta=.44, S E=.06)$. After controlling for maladaptive cognitions, inconsistent parenting still significantly predicted PIU $(\beta=.16, S E=.07)$. The application of the Sobel test to the mediation effect showed that a statistically significant change occurs upon the introduction of maladaptive cognitions into the mediation model $(z=2.75 ; p<.01)$. Nevertheless, inconsistent parenting ratings maintain prognostic value, which indicates a partial meditational role of maladaptive cognitions between inconsistent parenting and PIU ratings.

Table 3. Maladaptive Cognitions mediating the association between Inconsistent Parenting and Problematic Internet Use (PIU) $(n=307)$

\begin{tabular}{|c|c|c|c|c|c|}
\hline & $\boldsymbol{B}$ & $S D(B)$ & $\beta$ & $\boldsymbol{F}$ & $R^{2}$ \\
\hline 1. Inconsistent parenting $\rightarrow$ & & & & $12.11 * * *$ & .14 \\
\hline Maladaptive cognitions & .32 & .06 & $.29 * * *$ & & \\
\hline 2. Maladaptive cognitions $\rightarrow$ & & & & $17.80 * * *$ & .20 \\
\hline PIU & .50 & .06 & $.44 * * *$ & & \\
\hline 3. Inconsistent parenting $\rightarrow$ & & & & $6.833 * * *$ & .09 \\
\hline PIU & .36 & .07 & $.28 * * *$ & & \\
\hline 4. Predicting PIU & & & & $16.214 * * * *$ & .22 \\
\hline Inconsistent parenting & .20 & .07 & $.16 * *$ & & \\
\hline Maladaptive cognitions & .44 & .06 & $.39 * * *$ & & \\
\hline
\end{tabular}

$* * p<0,01 ; * * * p<0,001$. Note: included in the first step of each regression was age, gender and family economic situation. Sobel test confirmation of the partial mediation effect, $z=2.75 ; p<.01$. 


\section{Discussion}

The aim of this study was to examine the interrelationships of adolescent problematic internet use (PIU) and specific biological, social (parental) and cognitive factors. The design of the present study was based upon the theoretical framework presented by Brand and colleagues (Brand, et al., 2016), who have proposed the Interaction of Person-AffectCognition-Execution (I-PACE) model. This model is multi-faceted, including a vast number of interacting variables, and the present study was not with the intention of exploring all of the variables proposed in the I-PACE model. Rather, this study was with the intent of focusing upon three specific risk factors - hyperactivity ratings as an indication of biological/ genetic vulnerabilities; adolescent-rated parenting practices as reflecting the immediate social, familial environment in whch the adolescent is developing; and maldaptive cognitions, as proposed by Davis (2001) to be facilitating factors of problematic internet use.

The initial correlational analysis showed that hyperactivity ratings and maladaptive cognition ratings were positively and directly correlated with PIU. Also positively correlated with PIU were inconsistent parenting and harsh parenting practices. The final regression model, explaining $26 \%$ of the variance of PIU ratings showed the following to be significant predictors: hyperactivity and maladaptive cognitions. Maladaptive cognition ratings were shown to have a partial mediation effect on the association between inconsistent parenting and PIU ratings.

The positive association between hyperactivity and PIU ratings is consistent with that found in previous studies and supported by several meta-analyses investigating this relation (Carli, 2013; Wang, et al., 2017). One of the most prominent aspects of ADHD is a tendency to react impulsively, with difficulty in controlling one's behavioral and emotional reactions. This difficulty in self-control can help to explain why the adolescent with hyperactivity symptoms may have difficulty in self-imposing time limits in regard to internet use - and, as is known, an accelerated amount of time spent on the internet is facilitative of internet addiction. The "aversion for delayed rewards" which is characteristic of individuals with hyperactivity, may facilitate internet addiction because the internet does provide immediate rewards in the form of game points and other bonuses. Certainly, the immediacy of reward (whether in winning points in a game or making new online friends) available in the internet often far surpasses that which one encounters in real life.

Parental inconsistent discipline was found to be predictive of internet dependency, both directly and also as partially mediated by maladaptive cognition ratings. Inconsistent discipline indicates that parents may 
erratically fluctuate on whether or not to follow-through on certain threatened punishments, depending upon the parents' mood or the child's powers of persuasion. This implies that although the parent may be attempting to limit the adolescent's internet use, their efforts are inconsistent and thereby likely to be unsuccessful, and that inconsistent parenting can facilitate the development of maladaptive cognitions (Halgunseth, et al., 2013). In addition, a parent who is inconsistent in disciplining their child may also be inconsistent in providing emotional support and understanding, which would then implicate insecure parent-child attachment. It has been widely recognized from an attachment theory perspective (Bowlby, 1989; Scimmenti, et al., 2014) that insecure attachment relationships encourage the development of less adaptive cognitions of self and others - including a distrust of others. The results of the present study seem to support these theoretical propositions - faced with the social context of inconsistent parenting and insecure attachment, the adolescent has been more likely to develop a basic mistrust of others in his immediate environment, and therefore more likely to develop maladaptive cognitions such as "friends online are more trustable than those offline".

\section{Limitations and Future Direction}

Among the limitations of the present study is its cross-sectional nature. It would be meaningful to look at the interrelationships between the biological, emotional, cognitive and behavioral aspects of problematic internet use within a longitudinal study design, so as to examine possible unidirectional and bidirectional effects. Another possible limitation is that all ratings were self-reported by the adolescents. Although it has been variously argued that the adolescents' own perception of the behavior of him or herself and others within the near environment is more important than the perceptions of others (Jessor, et al., 2003), nevertheless the possibility of utilizing multiple sources in order to access ratings of behavior (e.g. particularly in regard to hyperactivity and parenting practices) would be of added benefit. Reliance solely upon the adolescents' reports may have inflated the correlations due to shared method variance.

Practical implications. The present study points to the value of considering the possible interrelationships of various risk-factors for PIU, and, subsequently, the value of considering preventive measures addressing these various aspects, and a combination of assessment measures and treatment methods when providing rehabilitation for adolescents suffering from internet addiction. When considering treatment for PIU, then it is important to consider various therapeutic techniques - for example, behavioral techniques in regard to minimizing hyperactivity symptoms, 
psychoeducational approaches with the parents in order to help them to understand the importance of emotional support and consistent structuring, and cognitive-behavioral approaches to address and restructure the adolescent's maladaptive cognitions.

\section{Conclusion}

Few studies to date have examined simultaneously biological, social and cognitive aspects as predictors of PIU. This study investigated hyperactivity, parenting practices and maladaptive cognitions, as examples of biological, social and cognitive processes which may pose as risk factors for the development of PIU. It was found that after controlling for age, gender and family economic status, regression analysis showed hyperactivity, inconsistent parenting and maladaptive cognitions to be predictive of PIU. Maladaptive cognitions were shown to partially mediate the effect between insonsistent parenting and PIU. The practical implications of our study include the necessity of considerting various preventive and treatment approaches for adolescents who are at risk of problematic internet use which hinders adaptive functioning in their daily academic and personal lives.

\section{References}

Anderson, C. A., Suzuki, K., Swing, E. L., Groves, C. L., Gentile, D. A., Prot, A., Lam, C. P., Sakamoto, A., Horiuchi, Y., Krahé, B., Jelic, M., Liuqing, W., Toma, R., Warburton, W. A., Zhang, X.-M., Tajima, S., Qing, F., \& Petrescu, P. (2017). Media violence and other aggression risk factors in seven nations. Personality and Social Psychology Bulletin, 43(7), 986-998.

Balan, R., Dobrean, A., Roman, G. D., \& Balazsi, R. (2017). Indirect effects of parenting practices on internalizing problems among adolescents: The role of expressive suppression. Journal of Child and Family Studies, 26, 40-47.

Bowlby, J. (1988). A Secure Base: Parent-Child Attachment and Healthy Human Development. Tavistock professional book. London: Routledge.

Brand, M., Young, K. S., \& Laier, C. (2014). Prefrontal control and internet addiction: atheoretical model and review of neuropsychological and neuroimagingfindings. Frontiers of Human Neuroscience, 8(375), 1-13.

Brand, M., Young, K. S., Laiera, C., Wölfling, K., \& Potenza, M. N. (2016). Integrating psychological and neurobiological considerations regarding the development and maintenance of specific Internet-use disorders: An Interaction of Person-Affect-CognitionExecution (I-PACE) model. Neuroscience and Biobehavioral Reviews, 71, 252-266.

Carli, V., Durkee, T., Wasserman, D., Hadlaczky, G., Despalins, R., Kramarz, E., Wasserman, C., Sarchiapone, M., Hoven, C. W., Brunner, R., et al. (2013). The association between pathological internet use and comorbid psychopathology: a systematic review. Psychopathology, 46(1), 1-13. 
Chen, Y. L., Chen, S. H., \& Gau, S. F. S. (2015). ADHD and autistic traits, family function, parenting style, and social adjustment for internet addiction among children and adolescents in Taiwan: A longitudinal study. Research in Developmental Disabilities, $39,20-31$.

Ciarrochi, J., Parker, P., Sahdra, B., Marshall, S., Jackson, C., Gloster, A. T., \& Heaven, P. (2016). The Development of compulsive internet use and mental health: A four-year study of adolescence, Developmental Psychology, 52(2), 272-283.

Davis, R. A. (2001). A cognitive-behavioral model of pathological internet use. Computers in Human Behavior, 17, 187-195.

Dong, G., Shen, Y., Huang, J., \& Du, X. (2013). Impaired error-monitoring function inpeople with internet addiction disorder: an event-related fMRI study. European Addiciton Research, 19, 269-275.

Ehlers, A., \& Clark, D. M. (2000). A cognitive model of posttraumatic stress disorder. Behavior Research and Therapy, 38, 319-345.

Eijnden, R. J. J. M. van den, Spijkerman, R., Vermulst, A. A., van Rooij, T. J., \& Engels, R. C. (2010). Compulsive internet use among adolescents: Bidirectional parentchild relationships. Journal of Abnormal Child Psychology, 38, 77-89.

Frick, P. J. (1991). The Alabama Parenting Questionnaire. Unpublished rating scale. University of Alabama.

Goodman, R. (1997). The Strengths and Difficulties Questionnaire: A research note. Journal of Child Psychology and Psychiatry, 38, 581-586.

Halgunseth, L. C., Perkins, D. F., Lippold, M. A., \& Nix, R. L. (2013). Delinquentoriented attitudes mediate the relation between parental inconsistent discipline and early adolescent behavior. Journal of Family Psychology, 27(2), 293-302.

Ho, R. C., Zhang, M. W. B., Tsang, T. Y., Toh, A. H., Pan, F., Lu, Y., \& Mak, K.-K. (2014). The association between internet addiction and psychiatric co-morbidity: A meta-analysis. BMC Psychiatr, 14, 183. Retrieved from http://dx.doi.org/10.1186/1471-244X-14-183.

Jessor, R.,Turbin, M. S., Costa, F. M., Dong, Q., Zhang, H. \& Wang, C. (2003). Adolescent Problem Behavior in China and the United States: A Cross-National Study of Psychosocial Protective Factors. Journal of Research on Adolescence, 13(3), 329-360.

Jusiene, R., Cesniene, I., \& Mordas, V. (2015). Problematic internet use and psychological well-being in adolescence. $17^{\text {th }}$ European Conference on Developmental Psychology. Braga, Portugal.

Kaess, M., Durkee, T., Brunner, R., Carli, V., Parzer, P., Wasserman, C., et al. (2014). Pathological internet use among European adolescents: Psychopathology and selfdestructive behaviours. European Child \& Adolescent Psychiatry, 23, 1093-1102.

Ko, C. H., Yen, J. Y., Yen, C. F., Lin, H. C., \& Yang, M. J. (2007). Factors predictive for incidence and remission of internet addiction in young adolescents: A prospective study. Cyberpsychology \& Behavior, 10, 545-551.

Li, D., Zhang, W., Li, X., Zhen, S., \& Wang, Y. (2010). Stressful life events and problematic internet use by adolescent females and males: A mediated moderation model. Computers in Human Behavior, 26, 1199-1207.

Meerkerk, G. J., Van Den Eijnden, R. J. J. M., Vermulst, A. A., \& Garretsen, H. F. L. (2009). The Compulsive Internet Use Scale (CIUS): Some psychometric properties. CyberPsychology \& Behavior, 12(1), 1-6. 
Miltuze, A., Bite, I., \& Sebre, S. (2012). Adolescent identity development, problematic internet use, computer game playing, dissociation, anxiety and sexual concerns. $15^{\text {th }}$ European Conference on Developmental Psychology, Proceedings, 251-257.

Nikolas, M. A., \& Burt, S. A. (2010). Genetic and environmental influences on and symptom dimensions of inattention and hyperactivity: A meta-analysis. Journal of Abnormal Psychology, 119(1), 1-17.

Park, S. K., Kim, J. Y., \& Cho, C. B. (2008). Prevalence of internet addiction and correlations with family factors among South Korean adolescents. Adolescence, 43(172), 895-909.

Pies, R. (2009). Should DSM-V designate "Internet addiction" a mental disorder? Psychiatry, 6, 31-37.

Schimmenti, A., Passanisi, A., Gervasi, A. M., Manzella, S., \& Famà, F. I. (2014). Insecure attachment attitudes in the onset of problematic internet use among late adolescents. Child Psychiatry and Human Development, 45, 588-595.

Sebre, S., Jusiene, R., Dapkevice, E., Skreitule-Pikse, I., \& Bieliauskaite, R. (2015). Parenting dimensions in relation to preschoolers' behavior problems in Latvia and Lithuania. International Journal of Behavioral Development, 39(5), 458-466.

Selfhout, M. H. W., Branje, S. J. T, Delsing, M., ter Bogt, T. F. M., \& Meeus, W. H. J. (2009). Different types of Internet use, depression, and social anxiety: The role of perceived friendship quality. Journal of Adolescence, 32(4), 819-833.

Shelton, K. K., Frick, P. J., \& Wootton, J. (1996). Assessment of parenting practices in families of elementary school-age children. Journal of Clinical Child Psychology, 25, 317-329.

Wang, B-Q., Yao, N-Q., Zhou, X., Liu, J., \& Lv, Z-T. (2017). The association between attention deficit/ hyperactivity disorder and internet addiction: a systematic review and metaanalysis. BMC Psychiatry, 17, 260. DOI 10.1186/s12888-017-1408-x.

Yen, J. Y., Yen, C. F., Chen, C. C., Chen, S. H., \& Ko, C. H. (2007). Family factors of internet addiction and substance use experience in Taiwanese adolescents. CyberPsychology \& Behavior, 10, 323-329.

Young, K. S. (1998). Internet addiction: The emergence of a new clinical disorder. CyberPsychology \& Behavior, 1, 237-244.

Zhang, H., Li, D., \& Li, X. (2015). Temperament and problematic internet use in adolescents: A moderated mediation model of maladaptive cognition and parenting styles. Journal of Child and Family Studies, 24, 1886-1897.

Zhou, Z., Yuan, G., \& Yao, J. (2012). Cognitive biases toward internet game-related pictures and executive deficits in individuals with an Internet game addiction. PLoS One, 7(11), e48961. Retrieved from http://dx.doi.org/10.1371/journal.pone.0048961. 